\title{
The Momentum of New Matter Replaces Dark Energy and Explains the Expansion of the Universe
}

\author{
Jarl-Thure Eriksson* \\ Åbo Akademi University, Finland \\ *Corresponding author: jarl-thure.eriksson@abo.fi \\ Received October 03, 2018; Revised November 06, 2018; Accepted November 19, 2018
}

\begin{abstract}
A new formulation of the Friedmann-Lemaitre-Robertson-Walker equation has been developed in a previous paper. The present study analyses an alternative approach to the cosmological constant $\Lambda$ from the perspective of the so called CBU model (Continuously Breeding Universe). Instead of having a cosmological constant responsible for the expansion, an excess pressure is boosted by a "bang factor" $\beta$. It is assumed that expansion is caused by the continuous addition of matter, due to, say, electron-positron fluctuations. Even if only a fraction of virtual pairs "stay alive", while the majority returns to nothingness, the phenomenon is vigorous enough to explain the expansion. The momentum change due to the inflow of new matter provides a force and accordingly a pressure explaining the expansion. An equation of said pressure is derived and then interpreted as the equivalent to the cosmological 'constant', $\Lambda_{\text {eq. }}$. The momentum pressure provides a perfect match to the dark energy density.
\end{abstract}

Keywords: general relativity, cosmological constant, Friedmann's equation, Robertson-Walker metric, dark energy

Cite This Article: Jarl-Thure Eriksson, "The Momentum of New Matter Replaces Dark Energy and Explains the Expansion of the Universe." International Journal of Physics, vol. 6, no. 5 (2018): 161-165. doi: 10.12691/ijp-6-5-4.

\section{Introduction}

In a paper published 1917 Albert Einstein introduced the cosmological constant $\Lambda$, [1], the purpose of which was to remove the dynamic instability of the universe as described by the General Theory of Relativity. Later Einstein regretted the additional term as the observations by Edwin Hubble showed that the universe was expanding. In principle Einstein could have envisaged the expansion, but for the wrong reason.

In order to expand, the system needs a "driving force", which is the reason why the cosmological constant has been reintroduced. In the Standard Cosmological Model $(\Lambda \mathrm{CDM}) \Lambda$ represents the dark energy content, also considered as the vacuum energy.

However, Einstein's paper of 1917 is interesting from some other points of view. He derives an equation for the curvature radius not very far from the Standard radius of the observable universe. He also speculates about the geometry of a space turning into itself.

In a recent paper the present author discusses the consequences that emerge, when certain well-founded assumptions are applied to the Friedmann equation, [2]. As a hypothesis the universe started from a quantum fluctuation, cf. Tryon, [3], and is expanding due a continuous creation of new matter, e.g. electron-positron fluctuations. The idea of continuous matter creation was first proposed by Fred Hoyle, [4], and Hermann Bondi \& Thomas Gold, [5], in the so called steady state theory. An addition of matter would keep the energy density constant in spite of expansion. In 1974 Paul Dirac wrote: "One might assume that nucleons are created uniformly throughout space, and thus mainly in intergalactic space. We may call this additive creation", [6].

If matter and accordingly energy is increasing, there must be a balancing mechanism in the form of negative energy. Alan Guth, [7], and Lawrence Krauss, [8], have been proponents of the idea, that all the energy in the universe sum up to zero, negative energy is provided by potential gravitational energy.

The model developed in Ref. [2] is called the Continuously Breeding Universe, CBU. The universe emerges from an initial electron-positron fluctuation. Acceleration is extremely high at the beginning, but decreases asymptotically to a very modest value of $\mathrm{g}_{\mathrm{u}}=1,066 \cdot 10^{-11} \mathrm{~m} / \mathrm{s}^{2}$ today. The model leads to a universe in good agreement with estimates based on satellite data. For instance, the age to proper length diagram, i.e. light-cone diagram, is in very good conformity with the Planck data of 2013. The result requires that the Hubble's constant is $\mathrm{H}_{\mathrm{o}}=68,24 \cdot 10^{3} \mathrm{~m} / \mathrm{s} / \mathrm{Mpc}$, just at the upper limit of the most recent results. The density of ordinary matter is $4,54 \cdot 10^{-28} \mathrm{~kg} / \mathrm{m}^{3}$, just within the limits given by NASA, [9].

One important result of the CBU model is a solution to the dark matter problem, which originates from the unexpected measurement results of galactic rotational speeds. The discrepancies are accurately explained by a Coriolis effect due to the accelerated expansion.

In the present article we will discuss some issues in the Einstein 1917 paper. Using the Robertson-Walker metric a 
comprehensive derivation of the Friedmann acceleration equation is accomplished. The Cosmological constant $\Lambda$ is replaced with a "bang-factor" $\beta$, it is shown that both alternatives are consistent with the original Friedmann-Lemaître-Robertson-Walker equation. Finally it is shown that dark energy is explained by the momentum pressure caused by the continuous addition of new matter.

\section{Hypotheses}

\subsection{Continuous Matter Creation}

Matter "created from the inside" is a logic explanation to the expansion of the universe. Several physicists have been proponents of this idea, as pointed out in the introduction. It is known for a fact that quantum fluctuations occurs constantly causing a quantum foam, cf. John Wheeler as quoted by Leonard Susskind, [10]. From reasons we don't know yet some of those virtual particles have time to annihilate into radiation and are incapable of returning to "nothingness". As a result there is a net increase of energy.

Hypothesis 1: The universe expands due to the addition of new matter. The positive energy is balanced by an equal amount of negative energy in the form of potential gravitational energy as reflected by the increase of space.

\subsection{Relation between Energy and Space}

In the years right after the publication of the General Theory of Relativity many implications were debated, one of which seemed to be a weird coincidence. If one squared the ratio of the radius of the universe $r_{u}$ and the estimated radius of the proton $r_{p}$, one would get a very large number corresponding to the ratio of the universe mass $\mathrm{M}_{\mathrm{u}}$ and the proton mass $\mathrm{m}_{\mathrm{p}}$ :

$$
\left(\frac{r_{u}}{r_{p}}\right)^{2}=\frac{M_{u}}{m_{p}} .
$$

Even if the motive to find a direct relation between the cosmos and the micro world was somewhat obscure, the fact that the energy of matter would be proportional to the radius squared was considered more than just a coincidence. This led Paul Dirac to define his Large Number Hypothesis (LNH) in 1937, [11].

Hypothesis 2: The total positive energy of the universe is proportional to the surface of a sphere having the radius $r_{u}$. We define

$$
W_{u}(t)=\pi b r_{u}^{2}(t)=4 \pi b r^{2}(t),
$$

where $r=r_{u} / 2$ is the radius of the observable universe, $b$ is a universal constant to be determined in section 4 .

\subsection{Size Dependent Gravitational Constant}

Mach's principle, after the Austrian physicist Ernst Mach, had a decisive impact on Einstein's thinking. According to the principle inertia is caused by the collective action of all masses in the universe. In 1953 Dennis Sciama, the mentor of Stephen Hawking, published a paper on the origin of inertia and concluded that

$$
\frac{G M_{u}}{r_{u} c^{2}}=k_{i},
$$

where $k_{i}$ is very close to 1 . Here we assume that $k_{i}=1$, which also has been suggested by Brans and Dicke in 1961, [12].

Assuming that the mass $\mathrm{M}_{\mathrm{u}}$ includes the equivalent mass of radiation, we have that $\mathrm{M}_{\mathrm{u}}=\mathrm{W}_{\mathrm{u}} / \mathrm{c}^{2}=4 \pi \mathrm{br}^{2} / \mathrm{c}^{2}$ where $\mathrm{W}_{\mathrm{u}}$ is the total positive energy. When this result is substituted into eq. (3), we obtain

$$
G=\frac{c^{4}}{2 \pi b r}
$$

Hypothesis 3: The gravitational constant $G$ is inversely proportional to the radius of the observable universe.

This is one of the most controversial issues in the CBU model. Astrophysicists of today strongly argue in favor of a constant G. However, it is logical that the gravitational force varies with the curvature (which is related to the radius $r$ ). A variable $G$ means that the Planck length $l_{P}$ is of the nuclear particle order in the early universe. An inflationary phase is not necessary.

\section{Peculiarities in Einstein's Paper on the Cosmological Constant}

According to the story Einstein regretted his 1917 paper, [1], by saying it was "his biggest blunder". It is uncertain if this was his real wording. He was discussing the paper with George Gamow, [13]. Probably they were using the German language, so most certainly Einstein did not use the word "blunder".

The paper includes several interesting features, whatever Einstein's opinion was. He derives an equation for what he calls the curvature radius, which later has been referred to as the Einstein radius

$$
R_{E}=\frac{c}{2} \sqrt{\frac{1}{\pi G \rho}},
$$

where $\rho$ is the average mass density of the universe.

If we assume that $\mathrm{k}_{\mathrm{i}}=4 / 3, \quad \mathrm{r}_{\mathrm{u}}=2 \mathrm{R}_{\mathrm{E}}$ and $M_{u}=32 \pi \cdot \rho \cdot R_{E}^{3} / 3$ and substitute them into eq (3), we will arrive at exactly the Einstein radius equation. Using the latest NASA estimate for the density of ordinary matter, $\rho=4,5 \cdot 10^{-28} \mathrm{~kg} / \mathrm{m}^{3}$, we obtain $\mathrm{R}_{\mathrm{E}}=4,88 \cdot 10^{26} \mathrm{~m}$, a value only $11 \%$ larger than the $\Lambda \mathrm{CDM}$ model radius of $4,396 \cdot 10^{26} \mathrm{~m}$.

The relevance of eq. (3) is emphasized by Einstein. Did he actually show that $\mathrm{G}$ is dependent of $\mathrm{r}\left(=\mathrm{R}_{\mathrm{E}}\right)$ ?

Another interesting detail is the remark "...als auch den Radius $\mathrm{R}$ des sphärischen Raumes und dessen Volumen $2 \pi^{2} R^{3 \prime}$ (...and also the radius $R$ and the volume $2 \pi^{2} R^{3}$ of the spherical space). Einstein uses the equation for the hyper-surface $\mathrm{S}_{4}$ of a $4 \mathrm{~d}$ space (cf. Wolfram MathWorld: Four-dimensional geometry), which also is the equation of the volume of a horn torus, i.e. a torus having the same major and minor radii (no central hole). The observer stands on the axis of revolution, the cross section is the observable universe, the horizon circumference is $2 \pi R$. The axis of revolution, length $2 \pi \mathrm{R}$, forms the curved space. 
The main purpose of Einstein's paper was the derivation of the cosmological constant

$$
\Lambda=\frac{1}{R^{2}} .
$$

The value appears to be circa 26 times smaller than the expansion requires, as we shall see in section 5 .

At the end of the paper Einstein states "The term $(\Lambda)$ is necessary only for the purpose of making possible a quasi-static distribution of matter, as required by the fact of the small velocities of the stars." An expanding universe was not on his agenda in 1917.

\section{Key Parameters}

At the initial moment a positron and an electron form the maiden universe. The "Eucledian" distance between the particles is $2 r_{i}$, but through the curved space the interaction distance is $\pi r_{i}$. The energy equilibrium is

$$
2 m_{e} c^{2}=G_{i} \frac{m_{e}^{2}}{\pi r_{i}}+\frac{e^{2}}{4 \pi \varepsilon_{0}\left(\pi r_{i}\right)},
$$

where $\mathrm{m}_{\mathrm{e}}$ and $\mathrm{e}$ are the electron mass and charge respectively. $G_{i}$ is obtained from eq. (3) given that $\mathrm{M}_{\mathrm{u}}=2 \mathrm{~m}_{\mathrm{e}}, \mathrm{r}=\mathrm{r}_{\mathrm{i}}$ and $\mathrm{k}=1$. We solve $\mathrm{r}_{\mathrm{i}}$

$$
r_{i}=\frac{e^{2}}{4 \pi \varepsilon_{0} m_{e} c^{2}(2 \pi-1)} .
$$

The value is $\mathrm{r}_{\mathrm{i}}=0,533381 \cdot 10^{-15} \mathrm{~m}$, a value of the same order of magnitude as the proton charge radius.

The energy constant of eq. (2) is

$$
b=\frac{2 m_{e} c^{2}}{4 \pi r_{i}^{2}}=8 \pi(2 \pi-1)^{2} \frac{\varepsilon_{0}^{2} m_{e}^{3} c^{6}}{e^{4}} .
$$

The value is $\mathrm{b}=0,458009 \cdot 10^{17} \mathrm{~J} / \mathrm{m}^{2}$.

From eq. (4) we are now able to determine the radius $r$ of the observable universe today. The value is

$$
\mathrm{r}_{0}=4,20578 \cdot 10^{26} \mathrm{~m},
$$

slightly smaller than the official value.

By substituting b from eq. (9) into eq. (4) we obtain an expression for $\mathrm{G}$ consisting of physical constants and the radius $r$

$$
G=\frac{e^{4}}{16 \pi^{2}(2 \pi-1)^{2} \varepsilon_{0}^{2} c^{2} m_{e}^{3}} \cdot \frac{1}{r} .
$$

\section{Expansion According to the Robertson-Walker Metric}

Einstein's field equation

$$
G_{\mu v}+\Lambda g_{\mu v}=\frac{8 \pi G}{c^{4}} T_{\mu v}
$$

has an analytic solution provided the energy-momentum tensor is isotropic and homogeneous. In the Robertson-Walker metric the solution takes the form

$$
\left(\frac{\dot{a}}{a}\right)^{2}=\frac{8 \pi G \rho}{3}-\frac{k c^{2}}{r_{\text {cur }}{ }^{2}}+\frac{1}{3} \Lambda c^{2},
$$

where $a$ is the scale factor, $\rho$ is the average mass density (radiation included), $\mathrm{k}$ is the curvature factor, $-1,0$ or 1 , $r_{\text {cur }}$ is the curvature radius and $\Lambda$ is the cosmological constant. If we assume that the universe is flat in space-time, $\mathrm{k}$ is zero.

Our next step is to derivate with respect to time

$$
\begin{gathered}
2 \dot{a} \ddot{a}=\frac{8 \pi}{3}\left(G a^{2} \dot{\rho}+2 G \rho a \dot{a}+\dot{G} \rho a^{2}\right) \\
+\frac{c^{2}}{3} \dot{\Lambda} a^{2}+\frac{2}{3} c^{2} \Lambda a \dot{a} .
\end{gathered}
$$

We can now write

$$
\frac{\ddot{a}}{a}=\frac{4 \pi G}{3}\left(\frac{a}{\dot{a}} \dot{\rho}+2 \rho+\frac{\dot{G}}{G} \rho \frac{a}{\dot{a}}\right)+\frac{c^{2}}{3}\left(\frac{1}{2} \dot{\Lambda} \frac{a}{\dot{a}}+\Lambda\right) .
$$

The equation differs from the accustomed form by taking into account time changes in $G$ and $\Lambda$.

In order to introduce the pressure responsible for the expansion we start with the 1. Law of Thermodynamics: $\mathrm{dW}+\mathrm{pdV}=0$. We derivate with respect to time

$$
\frac{d W}{d t}+p \frac{d V}{d t}=0
$$

The rate of volume expansion is

$$
\frac{d V}{d t}=k_{V} \frac{d}{d t}\left(r^{3}\right)=3 k_{V} r_{0}{ }^{3} a^{2} \dot{a}=3 V \frac{\dot{a}}{a},
$$

where $\mathrm{r}=\mathrm{ar}_{0}$ and $\mathrm{V}=\mathrm{k}_{\mathrm{V}} \cdot \mathrm{a}^{3} \mathrm{r}_{0}{ }^{3}$, the volume of the universe. $\mathrm{k}_{\mathrm{V}}$ is a volume factor, $32 \pi / 3$ for a sphere and $2 \pi^{2}$ for a $4 d$ hyper-surface $\mathrm{S}_{4}$. Here it is irrelevant which one we use.

The change of work is obtained from

$$
\frac{d W}{d t}=V \dot{\rho} c^{2}+\rho c^{2} \frac{d V}{d t}=V c^{2}\left(\rho+3 \rho \frac{\dot{a}}{a}\right) .
$$

When eqs. (16) and (17) are substituted into eq. (15), we obtain an expression containing $p$

$$
\dot{\rho} \frac{a}{\dot{a}}=-3\left(\rho+\frac{p}{c^{2}}\right) .
$$

When substituted into eq. (14) we get the complete version of the Friedmann-Lemaitre-Robertson-Walker (FLRW) equation

$$
\frac{\ddot{a}}{a}=-\frac{4 \pi G}{3}\left[\rho\left(1-\frac{\dot{G}}{G} \frac{a}{\dot{a}}\right)+3 \frac{p}{c^{2}}\right]+\frac{c^{2}}{3}\left(\frac{1}{2} \dot{\Lambda} \frac{a}{\dot{a}}+\Lambda\right) .
$$

From the relations $\mathrm{W}=\pi \mathrm{br}_{\mathrm{u}}{ }^{2}$ and $\mathrm{dW}=2 \pi b r_{\mathrm{u}}$. $\mathrm{dr}_{\mathrm{u}}=-\mathrm{pdV}$, we derive $\mathrm{p}=-\mathrm{b} / 2 \mathrm{r}_{\mathrm{u}}=-\mathrm{b} / 4 \mathrm{ar}_{\mathrm{o}}$. Further from eqs. (2) and (4) follows

$$
\dot{G}=-\frac{c^{4}}{2 \pi b r_{0}} \frac{\dot{a}}{a^{2}}, G=\frac{c^{4}}{2 \pi b r_{0} a}, \rho=\frac{3 b}{8 c^{2} r_{0} a} .
$$

These parameters as functions of the scale factor $a$ are substituted into eq. (19). It appears that the first term vanishes, which is a sign of an unstable state and the relevance of the initial hypotheses of section 2 . We are left with the following equation consisting only of $\Lambda$-terms 


$$
\frac{\ddot{a}}{a}=\frac{c^{2}}{3}\left(\frac{1}{2} \dot{\Lambda} \frac{a}{\dot{a}}+\Lambda\right) .
$$

We suggest the introduction of a new parameter $\beta$, which accounts for the additional pressure required to maintain the continuous expansion. This is an elegant way of dealing with the instability as compared to the cosmological constant, which is not considered a constant here. The extra pressure is provided by the continuous addition of matter. The alternative form of eq. (19) is

$$
\frac{\ddot{a}}{a}=-\frac{4 \pi G}{3}\left[\rho\left(1-\frac{\dot{G}}{G} \frac{a}{\dot{a}}\right)+3 \beta \frac{p}{c^{2}}\right] .
$$

When $\rho, G$ and $\dot{G}$ are substituted, the equation takes the form

$$
\ddot{a}=\frac{1}{2} \frac{c^{2}}{r_{0}^{2}} \frac{1}{a}(\beta-1) .
$$

The first time derivative of the scale factor $a$, i.e. expansion velocity, is solved by integration

$$
\dot{a}=\frac{c}{r_{0}} \sqrt{[\beta-1]} \sqrt{\ln \left(\frac{r}{r_{i}}\right)} .
$$

This is the Hubble parameter h. A further integration of eq. (23) leads to a relation between the time and the size of the universe. The integration, which is presented in Ref. [2], comprises an error function solution of the form

$$
t=D_{+} \frac{2 \cdot r}{c \sqrt{\beta-1}},
$$

where $D_{+}=D_{+}\left(\sqrt{ } \ln \left(r / r_{i}\right)\right)$ is the Dawson integral function. From eq. (24) we have

$$
\sqrt{\beta-1}=\frac{r_{0} H_{0}}{k_{h} c \sqrt{\ln \left(r_{0} / r_{i}\right)}}=0,315876,
$$

where $\mathrm{k}_{\mathrm{h}}=3,08567 \cdot 10^{22} \mathrm{~m} / \mathrm{Mpc}\left(\mathrm{H}_{0}=\mathrm{h}_{0} \mathrm{k}_{\mathrm{h}}\right)$. In Ref. [2] the Hubble parameter was estimated to $\mathrm{H}_{0}=68,24$ $\mathrm{km} / \mathrm{sMpc}$. We then have $\beta=1,099778$. The age of the present universe appears to be

$$
\mathrm{t}_{0}=4,544 \cdot 10^{17} \mathrm{~s} \text { or } 14,40 \mathrm{Gyr} \text {, }
$$

slightly larger than the Standard model age of 13,8 Gyr. We have to remember though, that there is a substantial difference between the beginning phases, Big Bang plus inflation against a "slow" but strongly accelerating initial start.

If we substitute eq. (23) into the energy equation (12), we find the relation between $\beta$ and $\Lambda$. Assuming that $\mathrm{k}=-1$ and that $\mathrm{r}_{\text {cur }}=\mathrm{r}$ we have

$$
\Lambda_{e q}=\frac{3}{r^{2}}\left[(\beta-1) \ln \left(r / r_{i}\right)-3 / 2\right]
$$

The value of $\Lambda_{\text {eq }}$ is $1,38 \cdot 10^{-52} \mathrm{~m}^{-2}$.

If eq. (26) and its time derivative are substituted into eq. (20), we will end up with eq. (22) thereby showing the consistency between eqs (12) and (22).

\section{Momentum Pressure Instead of Dark Energy}

The force caused by the momentum of new matter is obtained from

$$
F_{P}=\frac{d}{d t}(m v)=\frac{\partial m}{\partial t} v+m \frac{\partial v}{\partial t},
$$

where $\mathrm{v}$ is the velocity of expansion and $\mathrm{m}=2 \mathrm{~m}_{\mathrm{e}} \mathrm{N}_{\mathrm{e}+\mathrm{e}-\text {. }}$ From eq. (1) follows that the number of fluctuations is $\mathrm{N}_{\mathrm{e}+\mathrm{e}}=\left(\mathrm{r} / \mathrm{r}_{\mathrm{i}}\right)^{2}$. Utilizing the relations given in eqs (9) and (23) we end up with an equation for the momentum force

$$
F_{P}=8 \pi b r(\beta-1)\left[\ln \left(\frac{r}{r_{i}}\right)+\frac{1}{4}\right] .
$$

The energy provided by the momentum force is

$$
W_{P}=\int F_{P} d r=4 \pi b r^{2}(\beta-1)\left[\ln \left(\frac{r}{r_{i}}\right)-\frac{1}{4}\right] .
$$

In order to calculate the pressure $\mathrm{p}_{\mathrm{p}}$ we have to divide $\mathrm{W}_{\mathrm{P}}$ with the $4 \mathrm{~d}$ hyper-surface $\mathrm{S}_{4}=2 \pi^{2} \mathrm{r}^{3}$. The momentum pressure is

$$
p_{P}=\frac{2 b(\beta-1)}{\pi r}\left(\ln \frac{r}{r_{i}}-\frac{1}{4}\right) .
$$

In order to decide the nature of the curvature we change eq. (12) into a density equation

$$
\rho_{c r}=\rho_{O M}+\rho_{K}+\rho_{P},
$$

where

$$
\begin{gathered}
\rho_{c r}=\frac{3}{8 \pi G}\left(\frac{\dot{a}}{a}\right)^{2}=\text { critical density, } \\
\rho_{K}=-k \frac{3}{8 \pi G} \frac{c^{2}}{r_{c u r}{ }^{2}}=\text { 'curvature density'. }
\end{gathered}
$$

The density $\rho_{\mathrm{P}}$ is obtained by dividing eq. (30) with $\mathrm{c}^{2}$. $\rho_{\mathrm{OM}}$ represents ordinary matter including radiation.

When the right hand side of eq. (31) is divided by $\rho_{\mathrm{cr}}$ we obtain an expression familiar from the $\Lambda$ CDM model

$$
1=\Omega_{O M}+\Omega_{K}+\Omega_{P},
$$

where (obviously) $\Omega_{\mathrm{OM}}=\rho_{\mathrm{OM}} / \rho_{\mathrm{cr}}, \Omega_{\mathrm{K}}=\rho_{\mathrm{K}} / \rho_{\mathrm{cr}}$ and $\Omega_{\mathrm{P}}=\rho_{\mathrm{P}} / \rho_{\mathrm{cr}}$.

Table 1 shows some characteristics of the CBU and the $\Lambda C D M$ models, [14]. When the CBU numbers are applied to eq. (34) and we further assume that $r_{c u r}=r_{0}$ and $k=-1$, we end up with the following result

$$
\Omega_{\mathrm{OM}}+\Omega_{\mathrm{K}}+\Omega_{P}=1,00235 .
$$

Expansion accelerates because of a 2,35 per mille overshot in the critical density

Hereby it is convincingly established that the universe expands hyperbolically and that the curvature radius of the $3 \mathrm{~d}$ geometry of space is equal to the radius of the observable universe. It cannot be just a coincidence that the momentum pressure of eq. (30) perfectly balances the curvature and matter terms of eqs (31) and (34). 
It must, however, be remembered that the introduction of $\beta$ is an approximation, like $\Lambda$, and that a rigorous mathematical description of the early universe requires a deeper analysis of the origin of $\beta$ and its time dependence.

Considering that no form of matching of the models has been performed the agreement between key characteristics in Table 1 is very good. The momentum pressure leads to a higher "dark energy" density, but eliminates the need for dark matter.

Table 1. Comparison of the CBU and $\Lambda$ CDM models, [14] $\left(r=r_{0}\right)$

\begin{tabular}{|l|c|c|}
\hline & CBU & $\Lambda$ CDM \\
\hline Radius of the observable universe, $10^{26} \mathrm{~m}$ & 4,2058 & 4,3963 \\
\hline Hubble constant, $\mathrm{km} / \mathrm{sMpc}$ & 68,24 & 67,81 \\
\hline Critical density, $\rho_{\mathrm{cr}}, 10^{-27} \mathrm{~kg} / \mathrm{m}^{3}$ & 8,75 & 8,63 \\
\hline Density of ordinary matter, $\rho_{\mathrm{OM}}, 10^{-27} \mathrm{~kg} / \mathrm{m}^{3}$ & 0,453 & 0,422 \\
\hline Density of "dark energy", $\rho_{\mathrm{P}}$ and $\rho_{\mathrm{DE}}$ resp., $10^{-27} \mathrm{~kg} / \mathrm{m}^{3}$ & 7,405 & 5,97 \\
\hline Total amount of positive energy of the universe, $10^{71} \mathrm{~J}$ & 1,02 & 1,08 \\
\hline
\end{tabular}

\section{Conclusions}

The CBU model has a sound foundation in physics. Results are in good agreement with the present description of the universe. The evolutionary route differs from the traditional FLRW $\mathrm{t}^{2 / 3}$-scenario, but fits experimental supernova data as far back in time as observations reach. In a previous paper, [2], it was shown that the acceleration of the expansion explains kinematic discrepancies in galaxies due to the Coriolis effect, dark matter is not required. This means that the space within a galaxy also expands, a condition questioned by some astrophysicists. Empty space should not be mixed with an ether, it is not a substance.

Vacuum fluctuations are known as a fact. It is reasonable to regard the foam maintained by the fluctuations of virtual particles and antiparticles as the breeding source of new matter. According to the CBU model space is directly connected to the energy content, an increase of matter/energy results in an increase in size and the over all curvature radius. Space "bends" throughout due to the changing curvature. However, local concentrations of matter cause distortions, very small in comparison to the over all curvature radius.

It is shown that the cosmological 'constant' can be replaced by a factor $\beta$ describing the "inflow" of matter and thereby the primary origin of expansion. It seems more logical to presume a constant kick $(\beta)$ to the expansion pressure than to postulate a size and time independent cosmological constant. The replacement leads to a completely new picture of the universe's past. The CBU theory provides a continuous mathematical description of the emerging universe. Decaying acceleration of expansion, decreasing $G$ and a changing radius of curvature are factors making the interpretation of distant observations very complex.

The main finding of this paper is that dark energy has to be replaced by a kinetic energy constituent, the origin of which is the momentum of emerging matter.
Equilibrium requires that space is hyperbolically curved and that the curvature radius equals the radius of the observable universe. There are no alternative solutions to eq. (12). This is a plausible result. In his 1917 paper Einstein intuitively presumed that $r=r_{\text {cur }}$.

There are several open questions still to be answered. One concerns the mechanism responsible for the continuous breeding of new baryonic matter, the chain from positronelectron pairs to hydrogen atoms. Other questions are, how does the CMB fit into the model, what impact will an extremely high value of $\mathrm{G}$ in the past have on the redshift $z$ ? The answers may offer new avenues to explain the connection between gravity and quantum mechanics.

Summary of basic characteristics:

- The Universe is a complex emerging physical system.

- The Universe was initiated by a quantum fluctuation of an electrically charged elementary particle and its antiparticle.

- The Universe expands due to a continuous generation of elementary particle pairs.

- The positive energy of matter and radiation is the origin of space, outside the Universe there is no space, nothing.

- The positive energy of matter and radiation is balanced by an equal amount of negative potential gravitational energy.

- The space is curved into itself, the curvature radius is directly proportional to the size of the Universe.

- Stellar processes have no impact on the macroscopic expansion progress of the Universe.

\section{References}

[1] Einstein, A., Kosmologische Betrachtungen zur allgemeinen Relativitätstheorie. Sitzungsberichte der Preussischen Akad. d. Wissenschaften, 1917.

[2] Eriksson, J.-T., A modified model of the universe shows how acceleration changes galaxy dynamics, International Journal of Physics Vol 6, No. 2, pp. 38-46, 2018.

[3] Tryon, E. P., Is the universe a vacuum fluctuation?. Nature 247, pp. 396-397, 1973.

[4] Hoyle, F., A new model for the expanding universe, Mon. Not. Roy. Ast. Soc., 108, pp. 372-382, 1948.

[5] Bondi, H., Gold, T., The steady-state theory of the expanding universe, Mon. Not. Roy. Ast. Soc., 108, pp. 252-270, 1948.

[6] Dirac, P., Cosmological models and the Large Number hypothesis, Proc. R. Soc. Lond. A. 338, pp. 439-446, 1974.

[7] Guth, A. The Inflationary Universe: The quest for a new theory of cosmic origins, Perseus Books, 1997.

[8] Krauss, L., Universe from nothing, Simon \& Schuster, NY, 2012.

[9] NASA, Aeronautics and Space Administration, Universe 101, 2014.

[10] Susskind, L.,The Blach Hole War, Back Bay Books, NY, p.146, 2008.

[11] Dirac, P. A. M., The cosmological constants, Nature 139, 323, 1937.

[12] Brans, C., Dicke, R. H., Mach's principle and a relativistic theory of gravitation, Physical Review, 124, 3, pp. 925-935, 1961.

[13] Weinstein, G., George Gamow and Albert Einstein: Did Einstein say the cosmological constant was the "biggest blunder" he ever made in his life?, arXiv:1310.1033v1 [physics.hist-ph], 2013.

[14] Ade, P. A. R. et al. (Planck Collaboration), Planck 2015 results. XIII. Cosmological parameters, arXiv:1502.01589v3 [astroph.CO], 17 Jun 2016. 